\title{
A REMARK ON TSIRELSON'S STOCHASTIC DIFFERENTIAL EQUATION
}

\author{
M. Émery and W. Schachermayer
}

\begin{abstract}
Tsirelson's stochastic differential equation is called "celebrated and mysterious" by Rogers and Williams [16]. This note aims at making it a little more celebrated and a little less mysterious.

Using a deterministic time-change, we translate the study of Tsirelson's equation into the study of "eternal" Brownian motion on the circle. This allows us to show that the filtration generated by any solution of Tsirelson's equation is also generated by some Brownian motion (which, however, cannot be the Brownian motion driving the equation, because the equation has no strong solution).
\end{abstract}

\section{Introduction}

The so-called innovation problem is a remarkable phenomenon in the theory of filtered probability spaces; see for instance $\S 5.4$ of von Weizsäcker [24]. When the answer to the innovation problem is negative, some kind of creation of information occurs. This may happen in discrete, or continuous time (by discrete time, we refer to processes parametrized by $\mathbb{Z}$ ).

In discrete time, a paradigmatic example, well-known in ergodic theory, is obtained from an independent sequence $\left(U_{n}\right)_{n \in \mathbb{Z}}$ of random variables uniformly distributed on $\{-1,1\}$. Call $\left(\mathcal{G}_{n}\right)_{n \in \mathbb{Z}}$ the natural filtration of $U$ and $\left(\mathcal{F}_{n}\right)_{n \in \mathbb{Z}}$ the filtration generated by the "innovations" $V_{n}=U_{n-1} U_{n}\left(=U_{n} / U_{n-1}\right)$. Both $\mathcal{G}_{-\infty}$ and $\mathcal{F}_{-\infty}$ are trivial (by Kolmogorov's zero-one law), and both filtrations have $V_{n}$ as innovations: $V_{n}$ is independent of $\mathcal{F}_{n-1}$ (resp. $\mathcal{G}_{n-1}$ ), and, together with it, generates $\mathcal{F}_{n}$ (resp. $\mathcal{G}_{n}$ ). Yet, the filtration $\mathcal{G}$ strictly contains $\mathcal{F}$, because each random variable $U_{n}$ is independent of $\mathcal{F}_{\infty}$. More precisely, in the filtration $\mathcal{F}$, the process $U$ is observed up to multiplication by an unknown factor \pm 1 only. This example has been independently discovered by several authors; the earliest reference we know is Vershik [22], see also von Weizsäcker [24] and Exercise 4.12 in Williams [25].

In this example, the filtrations $\mathcal{G}$ and $\mathcal{F}$ are isomorphic, because they are generated by processes $\left(U_{n}\right)_{n \in \mathbb{Z}}$ and $\left(V_{n}\right)_{n \in \mathbb{Z}}$ with the same law.

In continuous time, there is an example very similar to the above discrete-time example. Replace the discrete time $\mathbb{Z}$ by the line $\mathbb{R}$ and the state space $\{-1,1\}$ by the circle $\mathbb{T}=\mathbb{R} / \mathbb{Z}$. Consider a $\mathbb{T}$-valued Brownian motion $\left(U_{t}\right)_{t \in \mathbb{R}}$ : each $U_{t}$ is uniformly distributed on $\mathbb{T}$ and $U$ moves Brownianly. For each $t \in \mathbb{R}$, call $\mathcal{G}_{t}$ the $\sigma$-algebra generated by all the past positions $U_{s}$ for $s \leqslant t$ and $\mathcal{F}_{t}$ the $\sigma$-algebra generated by all the past innovations $U_{t}-U_{s}$ for $s \leqslant t$. As above, each $U_{t}$ is independent of $\mathcal{F}_{\infty}$; this should be rather obvious on an intuitive level (and a formal proof will be provided below). As above, both filtrations $\mathcal{G}$ (generated by $U$ ) and $\mathcal{F}$ have trivial tail $\sigma$-fields at $-\infty$. By analogy with the previous example, a natural question is whether both filtrations are isomorphic. More precisely, it is easy to see that, after 
transforming the time-axis $\mathbb{R}$ into $(0, \infty)$ by the time-change $\log t \mapsto t, \mathcal{F}$ becomes the natural filtration of some real Brownian motion (first part of Proposition 3). The question becomes, is the time-changed filtration $\left(\mathcal{G}_{\log t}\right)_{t \geqslant 0}$ also generated by some Brownian motion? The next section will show that the answer is positive (second part of Proposition 3).

Another continuous-time example pertaining to the innovation problem is Tsirelson's stochastic differential equation $([21])$. This equation is of the form $d X_{t}=d B_{t}+g(t, X([0, t])) d t$, where $B$ is a Brownian motion and $g$ a bounded function of $t$ and of the past of $X$ up to $t$, given by some explicit formula involving fractional parts. Tsirelson has shown in [21] that solutions $X$ exist and all have the same law, but cannot be adapted to the natural filtration of $B$. Our third section will establish that, as far as filtrations are concerned, this situation is the same as the preceding one: given such a pair $(X, B)$ on the time-interval $[0, \infty)$, the natural filtrations of $X$ and $B$ become, after a deterministic time-change from $[0, \infty)$ to $[-\infty, \infty)$, those of a $\mathbb{T}$-valued Brownian motion and its innovations (Proposition 4). As a consequence, the natural (non time-changed) filtration of every solution $X$ to Tsirelson's stochastic differential equation is generated by some Brownian motion (Corollary 2).

This result should be appreciated in the context of the recent literature; in the remarkable paper [7], Dubins, Feldman, Smorodinsky and Tsirelson construct a variant of Tsirelson's equation whose solution $X$ generates a non-Brownian filtration. The question remained open, whether Tsirelson's original equation also has this property. As this note shows, the answer is no; in retrospect, this makes the - technically very involved - construction of [7] still more interesting. For further recent pertinent examples of Brownian and non-Brownian filtrations, we refer to $[11],[9],[10],[18],[20],[2],[5],[17],[23],[3],[8]$. Most of these examples feature the above-mentioned phenomenon of creation of information: there is a decreasing sequence of $\sigma$-fields, all of them containing some common information, but with trivial intersection. And Tsirelson's equation does exhibit this phenomenon, so much so, that it inspired Rogers and Williams to comment in [16]: "somehow, magically, this independent random variable has appeared from somewhere! Indeed, it really has appeared from thin air, because $[\ldots]$ it is not present at time 0 !"

Throughout this note, the usual hypotheses are in force: the probability spaces are complete, the filtrations contain all negligible events and are right-continuous.

\section{Circular Brownian motions}

The circle $\mathbb{R} / \mathbb{Z}$ is called $\mathbb{T}$; the symbol $\stackrel{1}{=}$ means equality modulo 1 (between two real numbers, or between a real number and an element of $\mathbb{T}$ ). For $x \in \mathbb{R}$ or $x \in \mathbb{T},\{x\}$ denotes the fractional part of $x$, that is, the real number $\{x\}$ such that $0 \leqslant\{x\}<1$ and $\{x\} \stackrel{1}{=} x$. If $U$ is a continuous, $\mathbb{T}$-valued process, we shall distinguish between the increment $U_{t}-U_{s}$, which takes its values in $\mathbb{T}$, and the real random variable $\int_{s}^{t} d U_{r}$, defined by the following three (lifting) conditions: it depends continuously on $t$, it vanishes for $t=s$, and $\int_{s}^{t} d U_{r} \stackrel{1}{=} U_{t}-U_{s}$. 
Definition. - Given a filtration $\mathcal{H}=\left(\mathcal{H}_{t}\right)_{t \in \mathbb{R}}$, a $\mathbb{T}$-valued process $\left(U_{t}\right)_{t \in \mathbb{R}}$ will be called a circular Brownian motion for $\mathcal{H}$ (abbreviated $\mathcal{H}$-CBM) if it is continuous and adapted to $\mathcal{H}$ and if for each $s \in \mathbb{R}$, the process $t \mapsto \int_{s}^{t} d U_{r}$, defined on the interval $[s, \infty)$, is a real-valued Brownian motion for the filtration $\left(\mathcal{H}_{t}\right)_{t \in[s, \infty)}$. (Equivalently, $U$ is a Markov process for $\mathcal{H}$, with transition probabilities the Brownian semigroup on the circle.)

If $\mathcal{H}$ equals the natural filtration $\mathcal{G}$ of $U$, we shall simply say that $U$ is a CBM. Note that this notion only depends on the law of $U$. It is easy to see that circular Brownian motions exist: as the uniform probability on $\mathbb{T}$ is invariant for the Brownian semigroup, a stationary, $\mathbb{T}$-valued Brownian motion with the uniform law at each fixed time is easy to construct. Uniqueness in law of CBM is less straightforward, but follows (among other possible proofs) from Proposition 1 below.

We have phrased the definition of a CBM $U$ with an arbitrary filtration $\mathcal{H}$ (instead of only considering the natural filtration $\mathcal{G}$ of $U$ ), as we shall encounter situations where $\mathcal{G}$ is strictly contained in another filtration $\mathcal{H}$. It then follows from the predictable representation property of (usual) Brownian motion that a CBM $U$ is an $\mathcal{H}$-CBM if and only if every $\mathcal{G}$-martingale is an $\mathcal{H}$-martingale.

Associated to a CBM $U$ are two filtrations: its natural filtration, generated by the past values $U_{t}$, and the innovation filtration, generated by the past increments $U_{t}-U_{s}$ or by their liftings $\int_{s}^{t} d U_{r}$ (which amounts to the same). Clearly, the innovation filtration is included in the natural filtration; Proposition 1 will show that it is always strictly smaller.

All CBM's have the same law, as shown by the next proposition, whose proof is borrowed from Proposition (6.13) of Stroock and Yor [19] (see also $\S$ IX.3 of [15] and $\S \mathrm{V} .18$ of $[16])$.

Proposition 1. - Let $U$ be a $C B M$, with innovation filtration $\mathcal{F}=\left(\mathcal{F}_{t}\right)_{t \in \mathbb{R}}$. For each $t \in \mathbb{R}$ the random variable $U_{t}$ is uniformly distributed on $\mathbb{T}$ and independent of the $\sigma$-field $\mathcal{F}_{\infty}$ (generated by all increments).

Proof. - Call $\mathcal{G}=\left(\mathcal{G}_{t}\right)_{t \in \mathbb{R}}$ the natural filtration of $U$ and for $p \in \mathbb{Z}$ denote by $e_{p}$ the character on $\mathbb{T}$ defined by $e_{p}(u)=\exp (2 \pi i p x)$ if $u \in \mathbb{T}, x \in \mathbb{R}$ and $u \stackrel{1}{=} x$. For $s<t, \int_{s}^{t} d U_{r}$ is independent of $\mathcal{G}_{s}$, with law $\mathcal{N}(0, t-s)$, so

$$
\begin{aligned}
\mathbb{E}\left[e_{p}\left(U_{t}\right) \mid \mathcal{G}_{s}\right] & =\mathbb{E}\left[e_{p}\left(U_{s}\right) e_{p}\left(U_{t}-U_{s}\right) \mid \mathcal{G}_{s}\right]=e_{p}\left(U_{s}\right) \mathbb{E}\left[e_{p}\left(U_{t}-U_{s}\right) \mid \mathcal{G}_{s}\right] \\
& =e_{p}\left(U_{s}\right) \mathbb{E}\left[\exp \left(2 \pi i p \int_{s}^{t} d U_{r}\right) \mid \mathcal{G}_{s}\right]=e_{p}\left(U_{s}\right) \exp \left[-\frac{1}{2} 4 \pi^{2} p^{2}(t-s)\right] .
\end{aligned}
$$

This implies

$$
\left|\mathbb{E}\left[e_{p}\left(U_{t}\right)\right]\right|=\left|\mathbb{E}\left[\mathbb{E}\left[e_{p}\left(U_{t}\right) \mid \mathcal{G}_{s}\right]\right]\right| \leqslant \mathbb{E}\left[\left|\mathbb{E}\left[e_{p}\left(U_{t}\right) \mid \mathcal{G}_{s}\right]\right|\right]=\exp \left[-\frac{1}{2} 4 \pi^{2} p^{2}(t-s)\right],
$$

and, by letting $s$ tend to $-\infty, \mathbb{E}\left[e_{p}\left(U_{t}\right)\right]=0$ for $p \neq 0$. As a consequence, by the Stone-Weierstraß theorem, $U_{t}$ has a uniform law on $\mathbb{T}$.

For each $s$, the $\sigma$-field $\mathcal{F}^{s}=\sigma\left(U_{t}-U_{s}, t \geqslant s\right)$ of increments after $s$ is independent of $\mathcal{G}_{s}$ and a fortiori of $U_{s}$. Thus, for $s<t$ and $p \neq 0$,

$$
\mathbb{E}\left[e_{p}\left(U_{t}\right) \mid \mathcal{F}^{s}\right]=\mathbb{E}\left[e_{p}\left(U_{s}\right) \mid \mathcal{F}^{s}\right] e_{p}\left(U_{t}-U_{s}\right)=\mathbb{E}\left[e_{p}\left(U_{s}\right)\right] e_{p}\left(U_{t}-U_{s}\right)=0,
$$

since we have just seen that $\mathbb{E}\left[e_{p}\left(U_{s}\right)\right]=0$. Now, $\bigvee_{s \in \mathbb{R}} \mathcal{F}^{s}=\mathcal{F}_{\infty}$, and, when $s \downarrow-\infty$, $\mathbb{E}\left[e_{p}\left(U_{t}\right) \mid \mathcal{F}^{s}\right] \rightarrow \mathbb{E}\left[e_{p}\left(U_{t}\right) \mid \mathcal{F}_{\infty}\right]$ by martingale convergence; so $\mathbb{E}\left[e_{p}\left(U_{t}\right) \mid \mathcal{F}_{\infty}\right]=0$ too, and $U_{t}$ is not only uniform, but also independent of $\mathcal{F}_{\infty}$. 
Definitions. - A regular time-change is an increasing (deterministic) bijection $a$ from $\mathbb{R}$ to $(0, \infty)$ such that both $a$ and its inverse $a^{-1}$ are absolutely continuous functions.

A filtration $\left(\mathcal{F}_{t}\right)_{t \geqslant 0}$ will be called Brownian if it is the natural filtration of some real Brownian motion $\left(B_{t}\right)_{t \geqslant 0}$ issued from the origin. (Only one-dimensional Brownian filtrations will be considered, so we simply call them Brownian.)

Proposition 2 And Definition. - Let $\left(\mathcal{F}_{t}\right)_{t \in \mathbb{R}}$ be a filtration indexed by $\mathbb{R}$. The following are equivalent:

(i) for some regular time-change a, the filtration $\left(\mathcal{F}_{t}^{\prime}\right)_{t \geqslant 0}$ defined by $\mathcal{F}_{t}^{\prime}=\mathcal{F}_{a^{-1}(t)}$ for $t>0$ and by right-continuity for $t=0$ is Brownian;

(ii) for every regular time-change a, the filtration $\left(\mathcal{F}_{t}^{\prime}\right)_{t \geqslant 0}$ defined by $\mathcal{F}_{t}^{\prime}=\mathcal{F}_{a^{-1}(t)}$ for $t>0$ and by right-continuity for $t=0$ is Brownian.

When these conditions are met, the filtration $\left(\mathcal{F}_{t}\right)_{t \in \mathbb{R}}$ is called Brownian.

For instance, if $\left(B_{t}\right)_{t \geqslant 0}$ is a real Brownian motion issued from the origin, the stationary Ornstein-Uhlenbeck process $\left(X_{t}\right)_{t \in \mathbb{R}}$ defined by $X_{t}=e^{-t / 2} B_{e^{t}}$ generates a Brownian filtration on $\mathbb{R}$; and conversely, every Brownian filtration on $\mathbb{R}$ is the natural filtration of such a stationary Ornstein-Uhlenbeck process.

Proof of Proposition 2. - We have to show (i) $\Rightarrow$ (ii); this reduces to checking that, if $a$ and $b$ are regular time-changes, the homeomorphism $\phi=a \circ b^{-1}$ from $(0, \infty)$ to itself has the following property: if $B$ is a Brownian motion on $[0, \infty)$, started at 0 , the process $X_{t}=B_{\phi(t)}$ generates a Brownian filtration. As $\phi$ and its inverse are absolutely continuous, the Lebesgue derivative $\psi$ of $\phi$ is almost everywhere defined and strictly positive. The process $X=B \circ \phi$ is a gaussian martingale, with quadratic variation $\phi(t)$. The martingale $\beta_{t}=\int_{0}^{t} \psi^{-1 / 2}(s) d X_{s}$ is a Brownian motion; as $X_{t}=\int_{0}^{t} \psi^{1 / 2}(s) d \beta_{s}$, both $X$ and $\beta$ generate the same filtration.

Definitions. - A chopping sequence is a sequence $\left(t_{k}\right)_{k \in \mathbb{Z}}$ of real numbers such that $t_{k}<t_{k+1}, \lim _{k \rightarrow-\infty} t_{k}=-\infty$ and $\lim _{k \rightarrow+\infty} t_{k}=+\infty$.

Given a chopping sequence $\left(t_{k}\right)_{k \in \mathbb{Z}}$, a chopped Brownian motion (respectively a $\mathbb{T}$-valued chopped Brownian motion) is a càdlàg process $Z$ whose restriction $Z^{k}$ to each interval $\left[t_{k}, t_{k+1}\right.$ ) is a real Brownian motion (respectively a $\mathbb{T}$-valued Brownian motion) started from 0 at time $t_{k}$, all the $Z^{k}$ 's being independent.

LEMMA 1. - The filtration generated by a chopped Brownian motion, or by a $\mathbb{T}$-valued chopped Brownian motion, is Brownian.

REMARK. - The converse also holds (but we shall not need it): every Brownian filtration indexed by $\mathbb{R}$ is the natural filtration of a chopped Brownian motion. More generally, if some càdlàg process $Z$ generates a Brownian filtration, every Brownian filtration is generated by a process with the same law as $Z$.

Proof of Lemma 1. - A $\mathbb{T}$-valued chopped Brownian motion $V$ clearly generates the same filtration as the real chopped Brownian motion $Z$ defined by $Z_{t}=\int_{t_{k}}^{t} d V_{s}$ for $t \in\left[t_{k}, t_{k+1}\right)$; so it suffices to prove the lemma for real processes.

The chopping sequence $\left(t_{k}\right)_{k \in \mathbb{Z}}$ and the chopped Brownian motion $Z$ are given. Introduce a sequence $\left(c_{k}\right)_{k \in \mathbb{Z}}$ of strictly positive constants such that $\sum_{k<0} c_{k}\left(t_{k+1}-t_{k}\right)<\infty$ and $\sum_{k \geqslant 0} c_{k}\left(t_{k+1}-t_{k}\right)=\infty$; define a regular time-change by 
and a process $\left(B_{t}\right)_{t>0}$ by $a(t)=\int_{-\infty}^{t} \sum_{k \in \mathbb{Z}} c_{k} 1_{\left(t_{k}, t_{k+1}\right]}(s) d s$

$$
B_{a(t)}=\sqrt{c_{k}} Z_{t}+\sum_{\ell \leqslant k} \sqrt{c_{\ell-1}} Z_{t_{\ell}-} \quad \text { for } t_{k} \leqslant t<t_{k+1} .
$$

By scaling, $B_{t}^{k}=B_{t}-B_{a\left(t_{k}\right)}=\sqrt{c_{k}} Z_{a^{-1}(t)}^{k}$ is a Brownian motion on the interval $\left[a\left(t_{k}\right), a\left(t_{k+1}\right)\right)$; and these Brownian motions $B^{k}$ are independent. So the series in the definition of $B$ is convergent and $B$ is a Brownian motion. As

$$
Z_{t}=\frac{1}{\sqrt{c_{k}}}\left(B_{a(t)}-B_{a\left(t_{k}\right)}\right) \quad \text { for } t_{k} \leqslant t<t_{k+1},
$$

the natural filtrations of $Z$ and $B$ are time-changed from each other, and $Z$ generates a Brownian filtration.

Proposition 3. - The natural filtration $\mathcal{G}$ and the innovation filtration $\mathcal{F}$ of a $C B M$ are Brownian.

Proof. - Let $U=\left(U_{t}\right)_{t \in \mathbb{R}}$ be a CBM, $\mathcal{G}$ its natural filtration, and $\mathcal{F}$ its innovation filtration. Choose any chopping sequence $\left(t_{k}\right)_{k \in \mathbb{Z}}$; for instance, $t_{k}=k$ is a possible choice. The chopped Brownian motion

$$
Z_{t}=\sum_{k \in \mathbb{Z}} \mathbb{1}_{\left[t_{k}, t_{k+1}\right)}(t) \int_{t_{k}}^{t} d U_{r}
$$

generates $\mathcal{F}$, so $\mathcal{F}$ is Brownian by Lemma 1 .

The proof for $\mathcal{G}$ is less straightforward. In fact, it involves a key idea of the present paper, a certain coupling lemma (for a vivid presentation of the use of coupling in the theory of Markov processes, see Diaconis [6]).

LEMmA 2. - If $\left(X_{t}\right)_{t \geqslant 0}$ is a $\mathbb{T}$-valued Brownian motion defined on some $\left(\Omega, \mathcal{A}, \mathbb{P},\left(\mathcal{H}_{t}\right)_{t \geqslant 0}\right)$, there exists a $\mathbb{T}$-valued Brownian motion $\left(Y_{t}\right)_{t \geqslant 0}$, defined on the same $\left(\Omega, \mathcal{A}, \mathbb{P},\left(\mathcal{H}_{t}\right)_{t \geqslant 0}\right)$, satisfying the following four properties:

(i) $Y_{0}=0 \in \mathbb{T}$;

(ii) $Y$ is independent of $X_{0}$;

(iii) both processes $\left(X_{t}\right)_{t \geqslant 0}$ and $\left(X_{0}, Y_{t}\right)_{t \geqslant 0}$ generate the same filtration;

(iv) calling $S$ the $\mathcal{H}$-stopping time $\inf \left\{t: X_{t}=Y_{t}\right\}$, one has $\mathbb{P}[S \geqslant t] \leqslant 1 /(4 t)$ and $Y=X$ on $\llbracket S, \infty \llbracket$.

Proof of Lemma 2. - Introduce the $\mathcal{H}$-stopping time $S=\inf \left\{t: X_{t}+X_{t}=X_{0}\right\}$. The process

$$
Y_{t}= \begin{cases}X_{0}-X_{t} & \text { if } t \leqslant S \\ X_{t} & \text { if } t \geqslant S\end{cases}
$$

is continuous by definition of $S$; it starts from 0 , is $\mathcal{H}$-adapted and verifies

$$
\int_{0}^{t} d Y_{s}=\int_{0}^{t}\left(-1_{\llbracket 0, S \rrbracket}+1_{\rrbracket S, \infty \llbracket}\right)(s) d X_{s},
$$

so it is a $\mathbb{T}$-valued $\mathcal{H}$-Brownian motion. As it starts from 0 , it is independent of $\mathcal{H}_{0}$, whence (ii). Replacing $\mathcal{H}$ by the natural filtration of $X$ shows that $\left(X_{0}, Y\right)$ is adapted 
to that filtration; to show (iii), it suffices to reconstruct $X$ from $X_{0}$ and $Y$. This is easy: $S$ is also the first time when $Y+Y=X_{0}$, and $X$ is equal to $X_{0}-Y$ up to $S$, and equal to $Y$ from $S$ on. Clearly, $S$ is the same as the one defined in (iv). Last, to establish the estimate $\mathbb{P}[S \geqslant t] \leqslant 1 /(4 t)$ in (iv), define

$$
T=\inf \left\{t:\left|\int_{0}^{t} d X_{s}\right|=\frac{1}{2}\right\} \text {. }
$$

When $t$ ranges from 0 to $T, X_{t}-X_{0}$ visits all points of one of the two arcs linking 0 and $\frac{1}{2}$ in the circle $\mathbb{T}$; so during this time-interval $\left(X_{t}-X_{0}\right)+\left(X_{t}-X_{0}\right)$ assumes all possible values on the circle, in particular the value $-X_{0}$. This implies $S \leqslant T$, whence the majoration $\mathbb{P}[S \geqslant t] \leqslant \mathbb{P}[T \geqslant t]$. Now the Brownian estimate $\mathbb{E}[T]=\mathbb{E}\left[\left(\int_{0}^{T} d X_{s}\right)^{2}\right]=( \pm 1 / 2)^{2}=1 / 4$ yields $\mathbb{P}[T \geqslant t] \leqslant \mathbb{E}[T] / t=1 /(4 t)$.

END OF THE PRoOf of Proposition 3. - To show that the natural filtration $\mathcal{G}$ of $U$ is Brownian, it suffices by Lemma 1 to exhibit a $\mathbb{T}$-valued chopped Brownian motion $V$ that generates $\mathcal{G}$. To this end, choose any chopping sequence $\left(t_{k}\right)_{k \in \mathbb{Z}}$ such that

$$
\sum_{k<0} \frac{1}{t_{k+1}-t_{k}}<\infty
$$

for instance, $t_{k}=k^{3}$ is a possible choice. For each $k$, Lemma 2 applied to the $\mathbb{T}$-valued Brownian motion $\left(U_{t}\right)_{t \geqslant t_{k}}$ provides us with a $\mathbb{T}$-valued Brownian motion $V^{k}$ defined on $\left[t_{k}, \infty\right)$, issued from 0 at time $t_{k}$, verifying condition (iii) of Lemma 2 , and equal to $U$ after some $\mathcal{G}$-stopping time $S_{k}$ such that $\mathbb{P}\left[S_{k}-t_{k} \geqslant t\right] \leqslant 1 /(4 t)$. We shall establish that the process $\left(V_{t}\right)_{t \in \mathbb{R}}$ equal to $V^{k}$ on $\left[t_{k}, t_{k+1}\right)$ is a $\mathbb{T}$-valued chopped Brownian motion and generates the filtration $\mathcal{G}$. Clearly, $V$ is $\mathcal{G}$-adapted.

To see that $V$ is a $\mathbb{T}$-valued chopped Brownian motion, we only have to show that the processes $\left(V_{t}^{k}\right)_{t \in\left[t_{k}, t_{k+1}\right)}$ are independent; it suffices to establish that $V^{k}$ is independent of $\mathcal{G}_{t_{k}}$. This can be obtained by writing, for a real-valued, bounded Borel functional $f$,

$$
\begin{aligned}
\mathbb{E}\left[f\left(V^{k}\right) \mid \mathcal{G}_{t_{k}}\right] & \stackrel{(1)}{=} \mathbb{E}\left[f \circ \phi_{k}\left(\left(U_{t}\right)_{t \geqslant t_{k}}\right) \mid \mathcal{G}_{t_{k}}\right] \stackrel{(2)}{=} \mathbb{E}\left[f \circ \phi_{k}\left(\left(U_{t}\right)_{t \geqslant t_{k}}\right) \mid U_{t_{k}}\right] \\
& =\mathbb{E}\left[f\left(V^{k}\right) \mid U_{t_{k}}\right] \stackrel{(3)}{=} \mathbb{E}\left[f\left(V^{k}\right)\right]
\end{aligned}
$$

where (1) stems from the fact that $V^{k}$ is a functional of $\left(U_{t}\right)_{t \geqslant t_{k}}$ (by Property (iii) of Lemma 2), (2) from the Markov property of $U$, and (3) from the independence of $V^{k}$ and $U_{t_{k}}$ (by Property (ii) of Lemma 2).

It remains to see that $V$ generates $\mathcal{G}$, or equivalently that $U$ is adapted to $V$. By Property (iii) of Lemma 2, there are some adapted Borel functionals $\psi_{k}$ such that

$$
\left(U_{t}\right)_{t \in\left[t_{k}, t_{k+1}\right)}=\psi_{k}\left(U_{t_{k}},\left(V_{t}\right)_{t \in\left[t_{k}, t_{k+1}\right)}\right) \quad \text { a. s. for each } k \text {. }
$$

(Adaptedness means that for $t \in\left[t_{k}, t_{k+1}\right)$, the restriction of $\psi_{k}(x, v)$ to $\left[t_{k}, t\right]$ is a function of $x$ and of the restriction of $v$ to $\left[t_{k}, t\right]$.) Using those $\psi$ 's, it is possible for each $k \in \mathbb{Z}$ to define inductively a $\mathbb{T}$-valued, càdlàg, $V$-adapted process $\left(U_{t}^{k}\right)_{t \in \mathbb{R}}$ by

$$
\begin{aligned}
U_{t}^{k} & =0 & & \text { for } t \in\left(-\infty, t_{k}\right), \\
\left(U_{t}^{k}\right)_{t \in\left[t_{k}, t_{k+1}\right)} & =\psi_{k}\left(V_{t_{k}-},\left(V_{t}\right)_{t \in\left[t_{k}, t_{k+1}\right)}\right), & & \\
\left(U_{t}^{k}\right)_{t \in\left[t_{\ell}, t_{\ell+1}\right)} & =\psi_{\ell}\left(U_{t_{\ell}-}^{k},\left(V_{t}\right)_{t \in\left[t_{\ell}, t_{\ell+1}\right)}\right) & & \text { for } \ell>k .
\end{aligned}
$$

(If the left-limit $U_{t_{\ell}-}^{k}$ is not defined, put for instance $U_{t}^{k}=0$ for $t \geqslant t_{\ell}$.) On the event $E_{k}=\left\{S_{k-1}<t_{k}\right\}$, one has $V_{t_{k}-}=U_{t_{k}-}=U_{t_{k}}$; by definition of $\psi_{k}$, this gives 
$U^{k}=U$ on $\left[t_{k}, t_{k+1}\right) \times E_{k}$. Now if, for some $\ell \geqslant k, U^{k}$ is equal to $U$ on the set $\left[t_{\ell}, t_{\ell+1}\right) \times E_{k}$, then $U_{t_{\ell+1}-}^{k}=U_{t_{\ell+1}-}=U_{t_{\ell+1}}$ on $E_{k}$ and so, by definition of $\psi_{\ell+1}$, $U^{k}$ agrees with $U$ on the next interval $\left[t_{\ell+1}, t_{\ell+2}\right) \times E_{k}$. Consequently, $U^{k}$ and $U$ are equal on $\left[t_{k}, \infty\right) \times E_{k}$.

Now take $t=t_{k+1}-t_{k}$ in the estimate $\mathbb{P}\left[S_{k}-t_{k} \geqslant t\right] \leqslant 1 /(4 t)$ and use the condition on the $t_{k}$ 's, to get $\sum_{k<0} \mathbb{P}\left[E_{k}^{\mathrm{c}}\right]<\infty$. The Borel-Cantelli lemma then says that $\lim \sup _{k \rightarrow-\infty} E_{k}^{\mathrm{c}}$ is negligible, and $E_{k}$ converges almost surely to $\Omega$ when $k \rightarrow-\infty$. So $\left[t_{k}, \infty\right) \times E_{k}$ tends to $\mathbb{R} \times \Omega$, and consequently $U^{k}$ converges (stationarily) to $U$. As each $U^{k}$ is $V$-adapted, so is also $U$.

REMARK (not used in the sequel). - When $\mathbb{T}$ is replaced by a compact, connected Riemannian manifold $\mathbb{M}$, it remains true that Brownian motion in $\mathbb{M}$ indexed by $\mathbb{R}$ has a Brownian filtration (but the innovation filtration can no longer be defined, unless $\mathbb{M}$ is endowed with some extra structure, e.g. Lie group or symmetric space). The definition of a Brownian filtration must of course be modified, with $d$-dimensional Bronian motions instead of real ones (where $d$ is the dimension of $\mathbb{M}$ ). We have not found a simple proof of this fact; M. Arnaudon establishes it in an appendix to this note by an argument using a mirror-coupling and some hypoelliptical diffusions. But the case when $\mathbb{M}$ is a $d$-dimensional sphere is much simpler (the mirror-coupling can be defined so as to avoid all cut-locus difficulties); as an illustration, we show here how that case can be dealt with, and refer to Arnaudon's appendix [1] for the general case.

It suffices to extend Lemma 2 to $\mathbb{M}$, the rest of the proof carries over almost verbatim. There are only two small differences: first, in (i) the point $0 \in \mathbb{T}$ has to be replaced with an arbitrary origin $O \in \mathbb{M}$; second, in (iv), any estimate of the form $\mathbb{P}[S \geqslant t] \leqslant f(t)$ will do, provided $f(t) \rightarrow 0$ when $t \rightarrow \infty$ (the condition $\sum_{k<0}\left(t_{k+1}-t_{k}\right)^{-1}<\infty$ in the proof will become $\left.\sum_{k<0} f\left(t_{k+1}-t_{k}\right)<\infty\right)$.

To prove the extended Lemma 2 , consider $\mathbb{M}$ as the subset of $\mathbb{R}^{d+1}$ made of all points at distance $R$ from the origin $0 \in \mathbb{R}^{d+1}$; for $x \in \mathbb{M}$ call $H_{x}$ the perpendicular bissector of the segment $[O x]$ and define the mirror-map $\Phi_{x}$ as the symmetry with respect to the hyperplane $H_{x} ; \Phi_{x}$ is an isometry of $\mathbb{M}$ exchanging $O$ and $x$ (when $x=O$, just take $\left.\Phi_{x}=\operatorname{Id}_{\mathbb{M}}\right)$. It then suffices to put $S=\inf \left\{t: X_{t} \in H_{X_{0}}\right\}$ (take $S=0$ if $\left.X_{0}=O\right)$ and $Y_{t}=\Phi_{X_{0}} X_{t}$ if $t \leqslant S, Y_{t}=X_{t}$ if $t \geqslant S$. This $Y$ is a $\mathbb{M}$-valued $\mathcal{X}$-Brownian motion satisfying (in $\mathbb{R}^{d+1}$ )

$$
Y_{t}=O+\int_{0}^{t}\left(\mathbb{1}_{\llbracket 0, S \rrbracket} \Phi_{X_{0}}+\mathbb{1}_{\rrbracket S, \infty \llbracket} \mathrm{Id}\right)\left(d X_{s}\right),
$$

whence properties (i), (ii) and (iii). To establish the estimate (iv), it suffices to show that, if $E$ denotes a fixed hemisphere and $T$ the first hitting time of the boundary $\partial E$ by a Brownian motion in $E$, then $\mathbb{P}^{x}[T \geqslant t]$ tends to 0 uniformly in $x \in E$ when $t \rightarrow \infty$. But the function $g(t, x)=\mathbb{P}^{x}[T \geqslant t]$ is continuous on $(0, \infty) \times \bar{E}$ (it is the solution to the heat equation $\partial g / \partial t=\frac{1}{2} \Delta g$ with boundary conditions $g=1$ for $t=0$ and $g=0$ on $\partial E$ ), and for each fixed $x$, it decreases to zero when $t \rightarrow \infty$; so uniformity in $x$ is a consequence of Dini's lemma.

Corollary 1. - Let $\left(\Omega, \mathcal{A}, \mathbb{P},\left(\mathcal{H}_{t}\right)_{t \in \mathbb{R}}\right)$ be a filtered probability space. There exists an $\mathcal{H}-C B M$ if and only if for some (or equivalently for every) regular time-change a, the filtration $\left(\mathcal{H}_{t}^{\prime}\right)_{t \geqslant 0}$ defined by $\mathcal{H}_{t}^{\prime}=\mathcal{H}_{a^{-1}(t)}$ admits an $\mathcal{H}^{\prime}$-Brownian motion (i.e., some $\mathcal{H}^{\prime}$-martingale is a Brownian motion). 
Proof. - If $U=\left(U_{t}\right)_{t \in \mathbb{R}}$ is an $\mathcal{H}$-CBM, its natural filtration $\mathcal{G}$ is Brownian by Proposition 3, and the time-changed filtration $\mathcal{G}^{\prime}$ is generated by a Brownian motion $B$ with $B_{0}=0$. As $U$ is a Markov process for $\mathcal{H}$, every $\mathcal{G}$-martingale is an $\mathcal{H}$-martingale, and, by time-change, every $\mathcal{G}^{\prime}$-martingale is an $\mathcal{H}^{\prime}$-martingale. So $B$ is an $\mathcal{H}^{\prime}$-Brownian motion.

Conversely, if $B$ is an $\mathcal{H}^{\prime}$-Brownian motion, call $\mathcal{G}^{\prime}$ the natural filtration of $B-B_{0}$ and notice that every $\mathcal{G}^{\prime}$-martingale is an $\mathcal{H}^{\prime}$-martingale. The time-change transforms $\mathcal{G}^{\prime}$ into the natural filtration $\mathcal{G}$ of some CBM $U$; as every $\mathcal{G}$-martingale is an $\mathcal{H}$-martingale, $U$ is also an $\mathcal{H}$-CBM.

\section{Tsirelson's stochastic differential equation}

In 1975, Tsirelson [21] has constructed the first example of a stochastic differential equation

$$
d X_{t}=d B_{t}+g\left[t,\left(X_{s}, s \leqslant t\right)\right] d t \quad X_{0}=0
$$

having the following properties: $B=\left(B_{t}\right)_{t \geqslant 0}$ is a Brownian motion, $g$ is a bounded, measurable function of the past $\left(X_{s}, 0 \leqslant s \leqslant t\right)$ of the solution $X=\left(X_{t}\right)_{t \geqslant 0}$, (T) has some (weak) solutions, but no solution $X$ can be adapted to the filtration generated by $B$.

Tsirelson's equation has been extensively studied; see for instance [4], [14], [19], [13], [26], [27], [12].

To define $g$, Tsirelson introduces a sequence $\left(t_{k}\right)_{k \leqslant 0}$ of instants verifying $t_{k}<t_{k+1}$ and $\lim _{k \rightarrow-\infty} t_{k}=0$. The function $g$ he considers is given by

$$
g\left[t,\left(X_{s}, s \leqslant t\right)\right]=\sum_{k<0}\left\{\frac{X_{t_{k}}-X_{t_{k-1}}}{t_{k}-t_{k-1}}\right\} \mathbb{1}_{\left(t_{k}, t_{k+1}\right]}(t)
$$

(recall that $\{x\}$ denotes the fractional part of $x$ ).

A solution to $(\mathrm{T})$ is a system $(\Omega, \mathcal{A}, \mathbb{P}, \mathcal{H}, B, X)$, where $(\Omega, \mathcal{A}, \mathbb{P}, \mathcal{H})$ is a filtered probability space, $B$ is an $\mathcal{H}$-Brownian motion started at 0 , and $X$ is $\mathcal{H}$-adapted and verifies $(\mathrm{T})$. Given such a solution, if $\mathcal{G}$ denotes the sub-filtration of $\mathcal{H}$ generated by $X$, then $B$ is clearly adapted to $\mathcal{G}$, so it is also a $\mathcal{G}$-Brownian motion, and $(\Omega, \mathcal{A}, \mathbb{P}, \mathcal{G}, B, X)$ is a solution too. The aim of this section is to establish that $\mathcal{G}$ is generated by some real Brownian motion started at the origin. This will be done by reducing the problem to the one addressed in Proposition 3; to do so, we shall need some notation.

Fix once and for all the sequence $\left(t_{k}\right)_{k \leqslant 0}$ and by this choice the function $g$. Define two functions $f$ and $\alpha$ on $(0, \infty)$ by

$$
f(t)=\sum_{k<0} \frac{1}{t_{k+1}-t_{k}} \mathbb{1}_{\left(t_{k}, t_{k+1}\right]}(t)+\mathbb{1}_{\left(t_{0}, \infty\right)}(t) \quad \alpha(t)=\int_{t_{0}}^{t} f(s)^{2} d s .
$$

As

$$
\int_{0}^{t_{0}} f(s)^{2} d s=\sum_{k<0} \frac{1}{t_{k+1}-t_{k}}=+\infty \quad \text { and } \quad \int_{t_{0}}^{\infty} f(s)^{2} d s=\int_{t_{0}}^{\infty} d s=+\infty,
$$

$\alpha$ is a homeomorphism from $(0, \infty)$ to $\mathbb{R}$; its inverse $a$ is a regular time-change. 
Proposition 4. - Fix the probability space $(\Omega, \mathcal{A}, \mathbb{P})$.

a) If, on $(\Omega, \mathcal{A}, \mathbb{P}),(\mathcal{H}, B, X)$ is a solution to $(\mathrm{T})$, define a $\mathbb{T}$-valued process $\left(U_{t}\right)_{t \in(0, \infty)} b y$

$$
U_{t} \stackrel{1}{=} \begin{cases}\frac{B_{t}-B_{t_{k}}}{t_{k+1}-t_{k}}+\frac{X_{t_{k}}-X_{t_{k-1}}}{t_{k}-t_{k-1}} & \text { for } t \in\left(t_{k}, t_{k+1}\right] \\ B_{t}-B_{t_{0}}+\frac{X_{t_{0}}-X_{t_{-1}}}{t_{0}-t_{-1}} & \text { for } t>t_{0}\end{cases}
$$

and time-change both $U$ and $\mathcal{H}$ by $\alpha$ : for $t \in \mathbb{R}$, set $\widetilde{U}_{t}=U_{a(t)}$ and $\widetilde{\mathcal{H}}_{t}=\mathcal{H}_{a(t)}$. The process $\widetilde{U}$ is a $\widetilde{\mathcal{H}}-C B M$.

b) Conversely, if $\left(\widetilde{\mathcal{H}}_{t}\right)_{t \in \mathbb{R}}$ is a filtration on $(\Omega, \mathcal{A}, \mathbb{P})$ and if $\left(\widetilde{U}_{t}\right)_{t \in \mathbb{R}}$ is an $\widetilde{\mathcal{H}}-C B M$, time-change them to a filtration $\left(\mathcal{H}_{t}\right)_{t \geqslant 0}$ and a process $\left(U_{t}\right)_{t>0}$ by $\mathcal{H}_{t}=\widetilde{\mathcal{H}}_{\alpha(t)}$ and $U_{t}=\widetilde{U}_{\alpha(t)} ;$ for $t>0$ set

$$
B_{t}=\int_{0}^{t} \frac{1}{f(s)} d U_{s} ; \quad X_{t}=B_{t}+\int_{0}^{t} \sum_{k<0}\left\{U_{t_{k}}\right\} \mathbb{1}_{\left.t_{k}, t_{k+1}\right]}(s) d s .
$$

Then $(\mathcal{H}, B, X)$ is a solution to $(\mathrm{T})$.

c) The two maps defined in a) and b) are inverse to each other; they establish a bijection between the solutions $(\mathcal{H}, B, X)$ to $(\mathrm{T})$ and the $C B M$ 's $(\widetilde{\mathcal{H}}, \widetilde{U})$.

d) Given a corresponding pair $(\mathcal{H}, B, X) \longleftrightarrow(\widetilde{\mathcal{H}}, \widetilde{U})$, call $\mathcal{G}$ the natural filtration of $X, \mathcal{F}$ that of $B, \widetilde{\mathcal{G}}$ that of $\widetilde{U}$ and $\widetilde{\mathcal{F}}$ the innovation filtration of $\widetilde{U}$. These filtrations are time-changed from one another:

$$
\widetilde{\mathcal{G}}_{t}=\mathcal{G}_{a(t)} \quad \mathcal{G}_{t}=\widetilde{\mathcal{G}}_{\alpha(t)} ; \quad \widetilde{\mathcal{F}}_{t}=\mathcal{F}_{a(t)} \quad \mathcal{F}_{t}=\widetilde{\mathcal{F}}_{\alpha(t)} .
$$

In particular, $\mathcal{H}$ is the natural filtration of $X$ if and only if $\widetilde{\mathcal{H}}$ is the natural filtration of $\widetilde{U}$.

REMARK. - This long statement can be summarized as follows. For fixed $(\Omega, \mathcal{A}, \mathbb{P})$, there exists a bijection between the solutions $(\mathcal{H}, B, X)$ to $(\mathrm{T})$ on the one hand and the pairs $(\widetilde{\mathcal{H}}, \widetilde{U})$ where $\widetilde{U}$ is a $\widetilde{\mathcal{H}}$-CBM on the other hand, with the following properties: The time-changes $a$ and $\alpha$ exchange the filtrations $\mathcal{H}$ and $\widetilde{\mathcal{H}}$; they also exchange the natural filtrations $\mathcal{G}$ of $X$ and $\widetilde{\mathcal{G}}$ of $\widetilde{U}$, as well as the natural filtration $\mathcal{F}$ of $B$ and the innovation filtration $\widetilde{\mathcal{F}}$ of $\widetilde{U}$.

So the process $X$ corresponds to $\widetilde{U}$ while $B$ corresponds to the innovations of $\widetilde{U}$. Remark that the triple $(\mathcal{H}, B, X)$ is redundant, for $B$ is a functional of $X$; consequently the proposition could be rephrased so as to state a correspondence between the pairs $(\mathcal{H}, X)$ and $(\widetilde{\mathcal{H}}, \widetilde{U})$; this would look more symmetric.

Proof of Proposition 4. - a) If $(\mathcal{H}, B, X)$ is a solution to $(\mathrm{T})$, for each $k<0$ one has

$$
\begin{aligned}
X_{t_{k+1}}-X_{t_{k}} & =B_{t_{k+1}}-B_{t_{k}}+\int_{t_{k}}^{t_{k+1}} g\left[t,\left(X_{s}, s \leqslant t\right)\right] d t \\
& =B_{t_{k+1}}-B_{t_{k}}+\left\{\frac{X_{t_{k}}-X_{t_{k-1}}}{t_{k}-t_{k-1}}\right\}\left(t_{k+1}-t_{k}\right) ;
\end{aligned}
$$

dividing both sides by $t_{k+1}-t_{k}$ and working modulo 1 to strip off the braces yields

$$
\frac{X_{t_{k+1}}-X_{t_{k}}}{t_{k+1}-t_{k}} \stackrel{1}{=} \frac{B_{t_{k+1}}-B_{t_{k}}}{t_{k+1}-t_{k}}+\frac{X_{t_{k}}-X_{t_{k-1}}}{t_{k}-t_{k-1}}
$$


Considered as elements of $\mathbb{T}$, the right-hand side is $U_{t_{k+1}}$ and the left-hand one is the limit of $U_{t}$ when $t$ tends to $t_{k+1}$ from above. This shows that the process $U$ is continuous at point $t_{k+1}$ and hence everywhere, and gives a meaning to $\int_{s}^{t} d U_{r}$.

For $s \leqslant t$ with $s$ and $t$ in the same interval $\left(t_{k}, t_{k+1}\right]$ or $\left(t_{0}, \infty\right)$, the definition of $U$ implies

$$
\int_{s}^{t} d U_{r}=\int_{s}^{t} f(r) d B_{r}
$$

By additivity, this formula remains valid for all pairs $s \leqslant t$ in $(0, \infty)$; notice that the right-hand side is an $\mathcal{H}$-martingale in $t$ on the interval $[s, \infty)$. By time-change, one gets, for $s \leqslant t$ in $\mathbb{R}$,

$$
\int_{s}^{t} d \widetilde{U}_{r}=\int_{a(s)}^{a(t)} f(r) d B_{r}
$$

and the right-hand side is an $\widetilde{\mathcal{H}}$-Brownian motion since $\int_{a(s)}^{a(t)} f^{2}(r) d r=t-s$. As $\widetilde{U}$

b) For each $s>0$, the process $\left(B_{t}\right)_{t \geqslant s}$ is a continuous $\mathcal{H}$-martingale, with quadratic variation

$$
\int_{s}^{t} \frac{1}{f^{2}(r)} d[U, U]_{r}=\int_{\alpha(s)}^{\alpha(t)} \frac{1}{f^{2}(\alpha(r))} d r=t-s
$$

hence a Brownian motion. Consequently, $\left(B_{t}\right)_{t \geqslant 0}$ is an $\mathcal{H}$-Brownian motion, starting at $B_{0}=0$.

Since $0 \leqslant\left\{U_{t_{k}}\right\}<1$, the integral in the definition of $X$ is convergent. For $k \leqslant 0$,

$$
\begin{aligned}
X_{t_{k}}-X_{t_{k-1}} & =B_{t_{k}}-B_{t_{k-1}}+\left\{U_{t_{k-1}}\right\}\left(t_{k}-t_{k-1}\right) \\
& =\int_{t_{k-1}}^{t_{k}} \frac{1}{f(r)} d U_{r}+\left\{U_{t_{k-1}}\right\}\left(t_{k}-t_{k-1}\right) \\
& =\left(t_{k}-t_{k-1}\right)\left[\int_{t_{k-1}}^{t_{k}} d U_{r}+\left\{U_{t_{k-1}}\right\}\right],
\end{aligned}
$$

so $\frac{X_{t_{k}}-X_{t_{k-1}}}{t_{k}-t_{k-1}} \stackrel{1}{=} U_{t_{k}}$, wherefrom $\left\{U_{t_{k}}\right\}=\left\{\frac{X_{t_{k}}-X_{t_{k-1}}}{t_{k}-t_{k-1}}\right\}$ and $X$ verifies $(\mathrm{T})$.

c) We first show that the composed map $(\mathcal{H}, B, X) \mapsto(\widetilde{\mathcal{H}}, \widetilde{U}) \mapsto\left(\mathcal{H}^{\prime}, B^{\prime}, X^{\prime}\right)$ is identity: $\left(\mathcal{H}^{\prime}, B^{\prime}, X^{\prime}\right)=(\mathcal{H}, B, X)$. That $\mathcal{H}^{\prime}=\mathcal{H}$ is trivial: two inverse time-changes cancel. Then, $B^{\prime}$ is defined by $d B_{t}^{\prime}=d U_{t} / f(t)$ and $U$ verifies $d U_{t}=f(t) d B_{t}$, giving $B^{\prime}=B$. Last, the right-continuity of $U$ at $t_{k}$ yields $U_{t_{k}} \stackrel{1}{=}\left(X_{t_{k}}-X_{t_{k-1}}\right) /\left(t_{k}-t_{k-1}\right)$, whence $d X_{t}^{\prime}=d B_{t}+g\left[t,\left(X_{s}, s \leqslant t\right)\right] d t=d X_{t}$, giving $X^{\prime}=X$.

Now the other way round: $(\widetilde{\mathcal{H}}, \widetilde{U}) \mapsto(\mathcal{H}, B, X) \mapsto\left(\widetilde{\mathcal{H}}^{\prime}, \widetilde{U}^{\prime}\right)$ is identity too. Again, $\widetilde{\mathcal{H}}^{\prime}=\widetilde{\mathcal{H}}$ is trivial. Time-change $\widetilde{U}$ and $\widetilde{U^{\prime}}$ to get a $U$ and a $U^{\prime}$. For $t \in\left[t_{k}, t_{k+1}\right)$ (with the convention $t_{1}=\infty$ ),

$$
U_{t}^{\prime} \stackrel{1}{=} \int_{t_{k}}^{t} f(s) d B_{s}+\frac{X_{t_{k}}-X_{t_{k-1}}}{t_{k}-t_{k-1}}=\int_{t_{k}}^{t} d U_{s}+\frac{X_{t_{k}}-X_{t_{k-1}}}{t_{k}-t_{k-1}}
$$

But we have seen in the proof of b) that $\frac{X_{t_{k}}-X_{t_{k-1}}}{t_{k}-t_{k-1}} \stackrel{1}{=} U_{t_{k}}$, so $U_{t}^{\prime}=U_{t}$.

d) As $X$ and $B$ are $\mathcal{G}$-adapted, the definition given in a), how to obtain $U$ from $B$ and $X$, shows that $U$ is $\mathcal{G}$-adapted. Conversely, the definitions given in b), how 
to obtain $B$ and $X$ from $U$, show that $X$ is adapted to the natural filtration of $U$. So $U$ has $\mathcal{G}$ as natural filtration; by time-change, this gives the relation between $\mathcal{G}$ and $\widetilde{\mathcal{G}}$.

The formulae $d B=(1 / f) d U$ and $d U=f d B$ show that the increments of $U$ generate the filtration $\mathcal{F}$; by time-change, this gives the relation between $\mathcal{F}$ and $\widetilde{\mathcal{F}}$.

Proposition 4 reduces the study of Tsirelson's equation to that of CBM's. Transferring to (T) what we know about CBM's gives the following statement (where only the last sentence is new):

Corollary 2. - Solutions $(\Omega, \mathcal{A}, \mathbb{P}, \mathcal{H}, B, X)$ to Tsirelson's equation exist. More precisely, on a given $\left(\Omega, \mathcal{A}, \mathbb{P},\left(\mathcal{H}_{t}\right)_{t \geqslant 0}\right)$, a solution $(B, X)$ exists if and only if there exists an $\mathcal{H}$-Brownian motion. The law of a solution $(B, X)$ depends only on the sequence $\left(t_{k}\right)_{k \leqslant 0}$. Given any solution, the natural filtration of $X$ is Brownian.

Proof. - If $(\mathcal{H}, B, X)$ is a solution, $B$ is an $\mathcal{H}$-Brownian motion. Conversely, if an $\mathcal{H}$-Brownian motion exists, the filtration $\left(\widetilde{\mathcal{H}}_{t}\right)_{t \in \mathbb{R}}$ defined by $\widetilde{\mathcal{H}}_{t}=\mathcal{H}_{a(t)}$ contains some CBM $\widetilde{U}$ by Corollary 1 and a solution $(\mathcal{H}, B, X)$ exists by Proposition 4.b.

The rest of the corollary is straightforward from Propositions 3 and 4 .

REMARKS. - a) If the reader does not care about CBM's and is only interested in knowing that the filtration of $X$ is Brownian, the proofs given above can be shortened.

First, in Proposition 4, only a) and d) are needed, so half of the computations can be dispensed with.

More important, a long detour we have taken can be bypassed. Our proof consisted in time-changing $(\mathrm{T})$ to get a CBM; and the proof that the filtration of a CBM is Brownian was done by time-changing back the CBM to work on $\mathbb{R}_{+}$(with a time-change provided by Lemma 1). By choosing in the proof of Proposition 3 the same (modulo the time-change) sequence $\left(t_{k}\right)_{k \leqslant 0}$ that is used to define $g$, it is possible to show directly that the filtration of $X$ is Brownian, with a proof quite similar to that of Proposition 3, that uses the time-changed CBM $U$ featuring in Proposition 4 a).

The reason for this detour was that we found it instructive, on an intuitive level, that the "mysterious" Tsirelson example may be one-to-one translated into Brownian motion on the circle, an object which to us does not seem mysterious at all.

b) After this work was completed, we learned that A. Vershik proved a long time ago the following (unpublished) result: When taken at the subdivision times $t_{n}$, a solution $X$ to $(\mathrm{T})$ generates a standard discrete filtration, a necessary condition for the continuous-time filtration to be Brownian. We find it striking that his (combinatorial) method of proof also involves coupling two (discrete) processes.

This standardness result was independently rediscovered by M. Malric (also unpublished). 


\section{References}

[1] M. Arnaudon. Appendice à l'exposé précédent : La filtration naturelle du mouvement brownien indexé par $\mathbb{R}$ dans une variété compacte. In this volume.

[2] M.T. Barlow, M. Émery, F.B. Knight, S. Song \& M. Yor. Autour d'un théorème de Tsirelson sur des filtrations browniennes et non browniennes. Séminaire de Probabilités XXXII, Lecture Notes in Mathematics 1686, Springer 1998.

[3] S. Beghdadi-Sakrani \& M. Émery. On certain probabilities equivalent to coin-tossing, d'après Schachermayer. In this volume.

[4] V. E. Beneš. Non existence of strong non-anticipating solutions to SDE's; implication for functional DE's, filtering and control. Stoch. Proc. Appl. 17, 243-263, 1977.

[5] B. De Meyer. Une simplification de l'argument de Tsirelson sur le caractère non-brownien des processus de Walsh. In this volume.

[6] P. Diaconis. From shuffling cards to walking around the building: An introduction to modern Markov chain theory. Documenta Mathematica. Extra volume ICM 1998. I 47-64, 1998.

[7] L. Dubins, J. Feldman, M. Smorodinsky \& B. Tsirelson. Decreasing sequences of $\sigma$-fields and a measure change for Brownian motion. Ann. Prob. 24, 882-904, 1996.

[8] M. Émery \& W. Schachermayer. Brownian filtrations are not stable under equivalent timechanges. In this volume.

[9] J. Feldman. $\varepsilon$-close measures producing non-isomorphic filtrations. Ann. Prob. 24, 912-915, 1996.

[10] J. Feldman \& M. Smorodinsky. Simple examples of non-generating Girsanov processes. Séminaire de Probabilités XXXI, Lecture Notes in Mathematics 1655, Springer 1997.

[11] J. Feldman \& B. Tsirelson. Decreasing sequences of $\sigma$-fields and a measure change for Brownian motion. II Ann. Prob. 24, 905-911, 1996.

[12] J. Kallsen. A stochastic differential equation with a unique (up to indistinguishability) but not strong solution. In this volume.

[13] J.-F. Le Gall and M. Yor. Sur l'équation stochastique de Tsirelson. Séminaire de Probabilités XVII, Lecture Notes in Mathematics 986, Springer 1983.

[14] R. S. Liptser \& A. N. Shiryaev. Statistics of Random Processes I. Springer, 1977.

[15] D. Revuz \& M. Yor. Continuous Martingales and Brownian Motion. Springer, 1991.

[16] L.C.G. Rogers and D. Williams. Diffusions, Markov Processes, and Martingales. Volume 2: Itô Calculus. Wiley, 1987.

[17] W. Schachermayer. On certain probabilities equivalent to Wiener measure, d'après Dubins, Feldman, Smorodinsky and Tsirelson. In this volume.

[18] M. Smorodinsky. Processes with no standard extension. Israel J. Math., to appear.

[19] D.W. Stroock \& M. Yor. On extremal solutions of martingale problems. Ann. Sci. École Norm. Sup. 13, 95-164, 1980.

[20] B. Tsirelson. Triple points: From non-Brownian filtrations to harmonic measures. GAFA, Geom. funct. anal. 7, 1096-1142, 1997.

[21] B. S. Tsirel'son. An example of a stochastic differential equation having no strong solution. Theor. Prob. Appl. 20, 427-430, 1975.

[22] A. M. Vershik. Approximation in measure theory. Doctor Thesis, Leningrad 1973. Expanded and updated english version: The theory of decreasing sequences of measurable partitions. St. Petersburg Math. J. 6, 705-761, 1995.

[23] J. Warren. On the joining of sticky Brownian motion. In this volume. 
[24] H. von Weizsäcker. Exchanging the order of taking suprema and countable intersections of $\sigma$-algebras. Ann. Inst. Henri Poincaré 19, 91-100, 1983.

[25] D. Williams. Probability with Martingales. Cambridge University Press, 1991.

[26] M. Yor. De nouveaux résultats sur l'équation de Tsirel'son. C. R. Acad. Sci., Paris, Sér. I 309, 511-514, 1989.

[27] M. Yor. Tsirel'son's equation in discrete time. Probab. Theory Relat. Fields 91, 135-152, 1992.

Michel Émery

Université Louis Pasteur et C.N.R.S.

I.R.M.A.

7 rue René Descartes

F 67084 Strasbourg Cedex

emery@math.u-strasbg.fr
Walter Schachermayer

Technische Universität Wien

Institut für Statistik, Wahrscheinlichkeitstheorie und Versicherungsmathematik

Wiedner Hauptstraße 8-10 / E 107-5

A 1040 Wien

walter.schachermayer@fam.tuwien.ac.at 